\title{
A study of molecular changes relating to energy metabolism and cellular stress in people with Huntington's disease: looking for biomarkers
}

\author{
Jolanta Krzysztoń-Russjan • Daniel Zielonka • \\ Joanna Jackiewicz • Sylwia Kuśmirek • Irena Bubko • \\ Aneta Klimberg • Jerzy T. Marcinkowski • \\ Elżbieta L. Anuszewska
}

Received: 26 June 2012 / Accepted: 4 October 2012 /Published online: 16 October 2012

(C) The Author(s) 2012. This article is published with open access at Springerlink.com

\begin{abstract}
Huntington's disease (HD) is a neurodegenerative disorder characterized by a progressive motor and cognitive decline and the development of psychiatric symptoms. The origin of molecular and biochemical disturbances in HD is a mutation in the HTT gene, which is autosomally dominantly inherited. The altered huntingtin protein is ubiquitously expressed in the CNS, as well as in peripheral tissues. In this study we measured the metabolism changes in gene transcription in blood of HD gene carriers (premanifest and manifest combined) versus 28 healthy controls. The comparison revealed statistically significant Global Pattern Recognition Fold Change (FC) for 6 mRNA transcripts, reflecting an increase of: $\mathrm{MAOB}(\mathrm{FC}=3.07 ; p=0.0005)$ which encodes an outer mitochondrial membrane-bound enzyme called monoamine oxidase type $\mathrm{B}$; TGM2 ( $\mathrm{FC}=1.8 ; p=0.02)$ encoding a transglutaminase 2 that mediates cellular stress; $\mathrm{SLC} 2 \mathrm{~A} 4$ (FC= $1.64 ; p=0.02$ ) solute carrier family 2 (facilitated glucose transporter) member 4; branched chain ketoacid dehydrogenase kinase $(\mathrm{BCKDK})(\mathrm{FC}=1.34 ; p=0.02)$; decrease of LDHA $(\mathrm{FC}=-1.16 ; p=0.03$ ) lactate dehydrogenase $\mathrm{A}$; and brainderived neurotrophic factor (BDNF) $(\mathrm{FC}=-2,11 ; p=0.03$ ). These distinguished changes coincided with HD progress.
\end{abstract}

J. Krzysztoń-Russjan $(\bowtie) \cdot J$. Jackiewicz $\cdot S$. Kuśmirek •

I. Bubko • E. L. Anuszewska

Department of Biochemistry and Biopharmaceuticals,

National Medicines Institute,

Chelmska 30/34 Str.,

00-725 Warsaw, Poland

e-mail: jrussjan@il.waw.pl

D. Zielonka $\cdot$ A. Klimberg $\cdot$ J. T. Marcinkowski

Chair of Social Medicine, Poznan University of Medical Science,

Rokietnicka 5C Str.,

60-806 Poznan, Poland
The analyses of gene transcription levels in sub-cohorts confirmed these changes and also revealed 28 statistically significant FCs of gene transcripts involved in ATP production and BCAA metabolism.

Keywords Huntington's disease - Energy metabolism · Transcriptomic biomarkers

\section{Introduction}

Huntington's disease (HD) is an autosomal dominant, neurodegenerative disorder with no effective treatment (The Huntington's Disease Collaborative Research Group 1993; Perez-De La Cruz and Santamaria 2007). The course of HD is slow and characterized by a gradual progression of motor, emotional and cognitive dysfunction leading to speech cessation, swallowing difficulties, walking problems and finally to the loss of independence and the whole organism devastation (Davies and Ramsden 2001; Ross and Tabrizi 2011). CAG triplet repeat expansion in the first exon of the $H T T$ gene results in an expansion of the polyglutamine (polyQ) tract in the Nterminus of the huntingtin protein (HTT) (Atwal et al. 2007); expansion above 35 polyQ results in the "mutant huntingtin" (mHTT). The unchanged polyQ tract is an active point of HTT in binding to other protein partners, but an elongated polyQ tract leads to the formation of nuclear and cytoplasmic aggregates, confers a gain-of-function as well as a loss-of-function of huntingtin and causes pathological changes on the cellular level inside and outside the CNS (Bjorkqvist et al. 2008; Sathasivam et al. 1999). Both the number of CAG repeats in the larger allele and epigenetic factors contribute to $\mathrm{HD}$ onset age and disease progression. 
Metabolic abnormalities in HD, including an impairment of cellular energy production and mitochondrial dysfunction, result in lower adenosine 5'-triphosphate (ATP) production (Chaturvedi et al. 2010; Cui et al. 2006; Liang and Ward 2006; Milakovic and Johnson 2005; Mochel et al. 2007, 2012; Weydt et al. 2006). Bioenergetics defects lead to weight loss and muscle wastage despite increased caloric intake in HD patients (Chaturvedi et al. 2010; Cui et al. 2006; Kosinski et al. 2007; Weydt et al. 2006). In addition, a systemic metabolic defect is closely related to the low level of branched chain amino acids (BCAA) in the serum or plasma of HD patients, which also results in early weight loss (Chaturvedi et al. 2010; Cui et al. 2006; Kosinski et al. 2007; Mochel et al. 2007, 2011; Weydt et al. 2006).

The activity of the peroxisome proliferator-activated receptor $\gamma(\operatorname{PPAR} \gamma)$ coactivator $1 \alpha(\mathrm{PGC}-1 \alpha)$ in human and mouse HD striatal and motor neurons is reduced due to the mHTT/ HTT related decrease of its transcription level. This reduction results in impairment of mitochondrial functions (Cui et al. 2006; St-Pierre et al. 2006; Weydt et al. 2006) as PGC-1 $\alpha$, a transcriptional co-factor, encoded by PPARGC1A gene, plays a key role regulating the expression of genes responsible for energy metabolism. It participates in energy homeostasis, adaptive thermogenesis, $\beta$-oxidation of fatty acids, and glucose metabolism by regulating the expression of mitochondrial OXPHOS genes and endogenous antioxidants (Puigserver et al. 2003). PGC-1 $\alpha$ also regulates the activity of histone deacetylase in muscles. Co-localization of HTT and PPARGC1A genes can influence transcription deregulation and is therefore considered to play an important role in HD pathogenesis (Kozlowski et al. 2010; Weydt et al. 2006). Mitochondrial malfunction in the basal ganglia of HD patients results in glucose metabolism reduction and lactate increase (Milakovic and Johnson 2005). Furthermore, biochemical studies revealed a reduced activity of several key components of oxidative phosphorylation, including complexes II, III, and IV of the electron transport chain in the mitochondria of striatal neurons in HD patients at an advanced stage of the disease (Beal et al. 1993; Browne and Beal 2004).

It is argued that the BCAA level decrease in the sera of HD subjects contributes to a BMI reduction and accelerates the disease progression (Mochel et al. 2007, 2011). BCAAs are involved in the mitochondrial intermediary metabolism as substrates in tricarboxilic acid (TCA), and their decreased level may indicate a systemic attempt to compensate for an early energy deficit in HD (Mochel et al. 2012). Therefore an identification of molecular changes associated with energy production and BCAA metabolism at the gene transcription level could identify biomarkers for the early detection of the disease as well as potential drug targets for effective therapies.

In this study we focused on energy metabolism pathways in blood cells in order to evaluate energy metabolism changes at the gene transcription level, using quantitative
Polymerase Chain Reaction (qPCR), of HD (pre-manifest and manifest) subjects compared to healthy subjects.

\section{Material and methods}

Subjects

Altogether 57 people were included in this study. The HD group - HDG $(n=29)$ consisted of pre-manifest HD $(n=6)$ and manifest HD $(n=23)$ subjects and the control group (CG) comprised healthy subjects $(n=28)$. Subject's key characteristics are presented in Table $1 \mathrm{a}$ and $\mathrm{b}$. All HDG individuals were confirmed carriers of the HTT gene mutation (ranged from 38 to $81 \mathrm{CAG}$ (mean $45, \mathrm{SD} \pm 15$ ) in the larger allele) Subjects were also characterized by their body mass index (BMI), calf circumference $(\mathrm{CC}$; $\mathrm{cm})$, to reflect condition of muscle mass, and a Mini Nutritional Assessment (MNA) evaluated the risk of malnutrition. HD motor symptoms were rated using the Unified Huntington Disease Rating Scale (UHDRS), and motor and functional impairment were evaluated using the Total Functional Capacity (TFC) assessment.

Subjects were identified using the REGISTRY Database of European Huntington's Disease Network. This study was approved by Bioethical Committee at Poznan University of Medical Science, Poland on September 03. 2009. Agreement No, 770/09. All human studies approved by this Agreement have been performed in accordance with Good Clinical Practice laid down in the Declaration of Helsinki.

\section{Blood sample collection}

Blood samples were collected from fasting (since 6.00 p.m. of the previous day) subjects between 8 and 10 a.m. General blood tests required $15 \mathrm{ml}$ and the Tempus collection tube (with RNA stabilizing buffer) (Applied Biosystems, Foster City, CA, USA) for total RNA isolation required $3 \mathrm{ml}$. To exclude disorders affecting metabolism, general blood tests of all participants were preformed for blood smear and morphology, CRP, ESR, triiodothyronine (FT3), tetraiodothyronine (FT4), TSH, cortisol, urea, creatinine, uric acid, cholesterol, protein fractions pattern and glucose levels.

\section{Total RNA extraction}

Total RNA was isolated using Tempus ${ }^{\mathrm{TM}}$ Spin RNA Isolation Kit (Applied Biosystems, Foster City, CA, USA) according to the manual instructions. Genomic DNA was eliminated by treating each sample with RNase-free DNase I (Fermentas Thermo Fisher Scientific Inc., Vilnius, Lithuania) according to the manual instructions. The concentration of isolated total RNA was calculated from absorbance at $260 \mathrm{~nm}$ with a BioPhotometer (Eppendorf, Hamburg, Germany), the purity 
Table 1 The study group and sub-cohorts clinical and genetic characteristics

\begin{tabular}{|c|c|c|c|c|}
\hline a) Study group and sub-cohorts & HDG $n=29$ & CG $n=28$ & b) HDG sub-cohorts & HDG \\
\hline Gender-cohort & & & CAG-cohort & \\
\hline Female & $n=16$ & $n=22$ & CAG number repeats & \\
\hline Male & $n=13$ & $n=6$ & Mean value (SD) & $45( \pm 15.0)$ \\
\hline Age-cohort & & & CAG1: $38-41$ & $n=9$ \\
\hline Age (years) & 46 & 40 & CAG2: $42-44$ & $n=10$ \\
\hline Mean value (SD)* & $( \pm 15.0)$ & $( \pm 15.0)$ & CAG3: $46-81$ & $n=10$ \\
\hline Age1: $\leq 35$ & $n=8$ & $n=12$ & TFC-cohort & \\
\hline Age2: $36-55$ & $n=15$ & $n=11$ & TFC (score in points) & \\
\hline Age $3: \geq 56$ & $n=6$ & $n=5$ & Mean value (SD) & $9.7( \pm 3.1)$ \\
\hline BMI-cohort & & & TFC1: $1-8$ & $n=7$ \\
\hline BMI $\left(\mathrm{kg} / \mathrm{m}^{2}\right)$ & 23.8 & 23.2 & TFC2: 9-10 & $n=9$ \\
\hline Mean value (SD) & $( \pm 4)$ & $( \pm 4.4)$ & TFC3: 11-13 & $n=13$ \\
\hline BMI1: up to 18.4 (underweight) & $n=2$ & $n=1$ & UHDRS-cohort & \\
\hline BMI2: 18.5-24.9 (standard) & $n=17$ & $n=19$ & UHDRS (score in points) & \\
\hline BMI3: 25.0-29.9 (overweight) & $n=6$ & $n=7$ & Mean value (SD) & $38.5( \pm 24.3)$ \\
\hline BMI4: 30.0-34.9 (obesity) & $n=4$ & $n=1$ & $\begin{array}{l}\text { UHDRS1: } 1- \\
8 \text { (presymptomatic) }\end{array}$ & $n=6$ \\
\hline CC-cohort & & & UHDRS2: $22-31$ & $n=5$ \\
\hline Calf circumference CC $(\mathrm{cm})$ & 36.0 & 36.7 & UHDRS3: $36-43$ & $n=8$ \\
\hline Mean value (SD) & $( \pm 3.6)$ & $( \pm 3.1)$ & UHDRS4: 45-55 & $n=6$ \\
\hline $\mathrm{CC} 1: 27-30$ & $n=4$ & $n=0$ & UHDRS5: 61-91 & $n=5$ \\
\hline $\mathrm{CC} 2: 31-35$ & $n=6$ & $n=12$ & HD disease duration (dd)-cohort & \\
\hline CC3: $36-38$ & $n=14$ & $n=10$ & HD duration period (years) & \\
\hline CC4 HDG/CG: $39-41 / 39-45$ & $n=5$ & $n=6$ & Mean value (SD) & $6.3( \pm 4.7)$ \\
\hline MNA-cohort & & & HD dd $1 ; 0$ (presymptomatic) & $n=6$ \\
\hline MNA (score in points) & 11.1 & 12.6 & HD dd $2 ; 1-5$ & $n=6$ \\
\hline Mean value (SD) & $( \pm 2.0)$ & $( \pm 1.3)$ & HD dd $3 ; 6-10$ & $n=12$ \\
\hline MNA1: 0-7 (malnourished) & $n=4$ & $n=2$ & HD dd $4 ; 11-15$ & $n=5$ \\
\hline MNA2: 8-11 (risk of malnutrition) & $n=10$ & $n=5$ & & \\
\hline MNA3: 12-14 ( normal nutritional status) & $n=15$ & $n=21$ & & \\
\hline
\end{tabular}

$S D^{*}$ standard deviation

was verified by optical density (OD) absorption ratio $\mathrm{OD}_{260 \mathrm{~nm}} /$ $\mathrm{OD}_{280 \mathrm{~nm}}$ between 1.60 and 1.8 , and $\mathrm{OD}_{260 \mathrm{~nm}} / \mathrm{OD}_{230 \mathrm{~nm}}$ ranging from 1.8 to 2.00 and the integrity was evaluated by electrophoresis on Gel - RNA Flash Gel System (Lonza Rockland Inc., Rockland, ME, USA). Total RNA subunits of $18 \mathrm{~S}$ and $28 \mathrm{~S}$ were observed on the gel and absence of smears indicating minimal degradation of the RNA, RNA template was suspended in RNase and DNase free water and stored at $-80{ }^{\circ} \mathrm{C}$ with RNase inhibitor (Fermentas Thermo Fisher Scientific Inc, Vilnius, Lithuania).

First strand cDNA synthesis

One microgram RNA was reverse-transcribed using the SYBR PrimeScript RT-PCR kit II (Takara Bio Inc., Otsu, Shiga, Japan) for first-strand cDNA synthesis using $2.5 \mu \mathrm{M}$ oligonucleotides primer and $5 \mu \mathrm{M}$ random hexamer for the priming method according to the manufacturer's recommendations. The first strand cDNA synthesis was started with the incubation of the transcription mixture at $37^{\circ} \mathrm{C}$ for $30 \mathrm{~min}$, to begin the reverse transcriptase reaction. Finally, the Prime Script Reverse Transcriptase was inactivated by heating the reaction mixture for $5 \mathrm{~s}$. at $85{ }^{\circ} \mathrm{C}$. Each RNA sample was controlled for genomic DNA contamination by incubation without the addition of reverse transcriptase into the cDNA synthesis mixture. All cDNA samples were stored at $-20{ }^{\circ} \mathrm{C}$ and diluted with RNase and DNase free water before being used as a template in the RT-qPCR (Reverse Transcriptase quantitative Real-Time Polymerase Chain Reaction) analysis.

qPCR

Quantitative PCR was performed using the MxPro 3005P apparatus (Stratagene, LaJolla, CA, USA) and the SYBR 
Premix Ex TaqTM II (Takara Bio Inc., Otsu, Shiga, Japan). The PCR Mix solution was previously made using the $974.68 \mu \mathrm{l}$ SYBR Premix, $1031.32 \mu \mathrm{l}$ RNase and DNase free water, and the $106 \mu$ liluted template contained $400 \mathrm{ng}$ of cDNA. The single PCR mix reaction volume was $20 \mu \mathrm{l}$. Gene expression analysis was conducted with the StellARray ${ }^{\mathrm{TM}}$ Gene Expression System (Lonza, Walkersville, MD, USA), a type of qPCR array, with previously optimized primer concentrations applied into the 96 wells plates. Thermocycling conditions were set as follows: an initial PCR mix activation was for $2 \mathrm{~min}$. at $50^{\circ} \mathrm{C}$, the next polymerase activation step was for $15 \mathrm{~s}$. at $95^{\circ} \mathrm{C}$, then 40 cycles of $15 \mathrm{~s}$. at $95{ }^{\circ} \mathrm{C}$ (template denaturation) and $1 \mathrm{~min}$. at $60{ }^{\circ} \mathrm{C}$ (for annealing and elongation). A dissociation protocol with a gradient from $95{ }^{\circ} \mathrm{C}$ to $55^{\circ} \mathrm{C}$ and to $95^{\circ} \mathrm{C}$ was used for each primer pair to verify the specificity of the RT-qPCR reaction and the absence of primer-dimer control. In addition, each PCR reaction included a reverse transcription negative control to check for potential genomic DNA contamination. Reagent contamination was also detected by a reaction mix without template.

The panel of 95 genes, including normalizers, was previously selected for this study. The gene panel focused on several pathways involved in energy metabolism (ATP production) such as: glycolysis/Krebs cycle, electron transport chain, mitochondria biogenesis and construction, BCAA metabolism, transcription factors related to energy production, cell cycle, cell stress/immune response and other cellular pathways (Table 2).

An initial analysis was performed using the MxPro 3005P system software for determining the $\mathrm{Ct}$ (cycle threshold) value after threshold assessment. The relative transcription level was calculated based on $\Delta \Delta \mathrm{Ct}$ type of analysis using the $2^{-\Delta \Delta \mathrm{Ct}}$ method in order to Fold Change (FC) value determination between compared groups. The data obtained after qPCR was analyzed by Global Pattern Recognition, statistical software (GPR; https://array.bhbio. com/BHB/GUI/AP/GPR.aspx). Normalization to $18 S$ rRNA, was used as internal control, and 9 additional genes normalizers simultaneously distinguished by GPR software analysis were performed for each gene tested (Akilesh et al. 2003). The GPR algorithm allows the detection of significant changes in gene expression patterns by comparing the expression of each gene to every other gene in the array. Significant changes are identified and ranked providing relative normalization in qPCR experiments.

Statistical methods and calculations

A normal distribution of analytical results was verified and confirmed. Then the t-Student's test for differences between HDG and CG was preformed. The GPR was used to determine the gene transcript level changes for the HDG with reference to the $\mathrm{CG}$ (calibrator) using cycle threshold $(\mathrm{Ct})$ values obtained for all the genes tested. The GPR FC value was calculated for each gene tested. FC with $p \leq 0.05$ was defined as statistically significant. For all the genes tested in the distinguished cohorts, several comparisons were performed in order to screen for statistical significance in FCs. The statistically significant FCs across sub-cohorts were analyzed by the Kruskal Wallis $\left(\mathrm{Chi}^{2}\right)$ test and compared using the Mann-Whitney U test - (Table 3a-i). The Spearman's coefficient was calculated to verify the correlation for analytical results with gene transcription level changes expressed by FC (in the sub-cohorts), (Tables 4 and 5). SPSS v. 19.0 software (SPSS Inc., USA) and Statistica v. 9. software, (StatSoft Inc, USA) were used for statistical calculations.

\section{Results}

\section{Analytical tests}

Comparison between analytical test results in both HDG and CG was performed. Apart from cholesterol, HDL and LDL levels in HDG, and HDL in CG, all other values of the analytical test results in both groups did not exceed the range of reference values. The cholesterol and LDL levels were slightly higher ( 210.1 and 131.6 respectively) in HDG than in the control (198.7 and 119.4 respectively), but not significant in the t-Student test. Total proteins, $\beta 2$ fraction levels, alfa-1 globulins level and FT3 level were significantly different between the CG and HDG, but were within the range of reference values. Positive significant correlations in the HGD among the HGB, HCT, ESR, CRP MNA, MHCH levels as well as lymphocytes amount and BMI were found. In the CG, a significant positive correlation between CC and WBC and also BMI and CRP was obtained (Table 5).

The relations between HD defining parameters, anthropometric tests and MNA score

Correlations among clinical HD defining parameters, such as: TFC, disease duration and CAG repeats number in the larger allele and BMI, CC and MNA score were calculated based on the Spearman's coefficient factor analysis (Table 4). The statistically significant positive correlations were found between the age of subjects and the age of HD onset $(R=0.718 ; p<$ $0.01)$ and between the calf circumference and TFC score $(R=$ $0.386 ; p=0.039)$ and also between MNA and TFC score $(R=$ $0.525 ; p=0.003)$. Results of these correlations indicate that as HD progresses, the calf circumference and MNA score decreases. A negative correlation was only found between the age of HD subjects and the number of CAG repeats in the larger allele $(R=-0.551 ; p=0.002)$. 
Table 2 A list of genes tested in this study

\begin{tabular}{llll}
\hline & Gene ID & Gene Symbol & Function \\
\hline \multicolumn{2}{l}{ Glycolysis/Krebs cycle } & & \\
$\mathbf{1}$ & $\mathbf{5 0}$ & ACO2 $^{\text {a }}$ & Aconitase 2, mitochondrial \\
2 & 226 & $A L D O A$ & Aldolase A, fructose-bisphosphate \\
3 & 2597 & $G A P D H$ & Glyceraldehyde-3-phosphate dehydrogenase \\
4 & 3417 & $I D H 1$ & Isocitrate dehydrogenase 1 (NADP+) \\
$\mathbf{5}$ & $\mathbf{3 9 3 9}$ & LDHA & Lactate dehydrogenase A \\
6 & 4967 & $O G D H$ & Oxoglutarate (alpha-ketoglutarate) dehydrogenase (lipoamide) \\
7 & 5160 & $P D H A 1$ & Pyruvate dehydrogenase (lipoamide) alpha 1 \\
8 & 5163 & $P D K 1$ & Pyruvate dehydrogenase kinase, isozyme 1 \\
9 & 5223 & $P G A M 1$ & Phosphoglycerate mutase 1 (brain)
\end{tabular}

Electron transport chain/ATP production/mitochondria biogenesis and construction

$\begin{array}{llll}\mathbf{1 0} & \mathbf{4 8 8} & \boldsymbol{A T P 2 \boldsymbol { A }} & \begin{array}{l}\text { ATPase, Ca++ transporting, cardiac muscle, slow twitch } 2 \\ \text { Cytochrome c oxidase subunit IV isoform 1 }\end{array} \\ \mathbf{1 1} & \mathbf{1 3 2 7} & \boldsymbol{C O X} 4 \boldsymbol{I 1} & \text { COX4 neighbor } \\ 12 & 10328 & C O X 4 N B & \text { Cytochrome c oxidase subunit II } \\ \mathbf{1 3} & \mathbf{4 5 1 3} & \boldsymbol{C O X 2} & \text { Carnitine palmitoyltransferase 1A } \\ 14 & 1374 & C P T 1 A & \text { Monoamine oxidase B } \\ \mathbf{1 5} & \mathbf{4 1 2 9} & \boldsymbol{M A O B} & \text { Succinate dehydrogenase complex, subunit B, iron sulfur (Ip) } \\ 16 & 6390 & S D H & \text { Uncoupling protein 2 (mitochondrial, proton carrier) } \\ \mathbf{1 7} & \mathbf{7 3 5 1} & \boldsymbol{U C P 2} & \text { Uncoupling protein 3 (mitochondrial, proton carrier) } \\ 18 & 7352 & U C P 3 & \end{array}$

BCAA metabolism

$\begin{array}{lll}19 & 27034 & \text { ACAD } 8 \\ 20 & 4329 & \text { ALDH6A1 } \\ 21 & 549 & \text { AUH } \\ \mathbf{2 2} & \mathbf{5 8 7} & \text { BCAT2 } \\ \mathbf{2 3} & \mathbf{5 9 3} & \text { BCKDHA } \\ \mathbf{2 4} & \mathbf{5 9 4} & \text { BCKDHB } \\ \mathbf{2 5} & \mathbf{1 0 2 9 5} & \text { BCKDK } \\ 26 & 1629 & D B T \\ 27 & 1738 & D L D \\ 28 & 11112 & H I B A D H \\ 29 & 56922 & M C C C 1 \\ 30 & 64087 & M C C C 2\end{array}$

Acyl-Coenzyme A dehydrogenase family, member 8

Aldehyde dehydrogenase 6 family, member A1

AU RNA binding protein/enoyl-Coenzyme A hydratase

Branched chain aminotransferase 2, mitochondrial

Branched chain keto acid dehydrogenase $\mathrm{E}$, alpha polypeptide

Branched chain keto acid dehydrogenase E1, beta polypeptide

Branched chain ketoacid dehydrogenase kinase

Dihydrolipoamide branched chain transacylase E2

Dihydrolipoamide dehydrogenase, E3

3-hydroxyisobutyrate dehydrogenase

Methylcrotonoyl-Coenzyme A carboxylase 1 (alpha)

Methylcrotonoyl-Coenzyme A carboxylase 2 (beta)

Transcription factors related to the energy production

$\begin{array}{lll}\mathbf{3 1} & \mathbf{1 0 8 9 1} & \text { PPARGC1A } \\ \mathbf{3 2} & \mathbf{2 3 0 8 2} & \text { PPRC1 } \\ 33 & 5465 & \text { PPARA } \\ 34 & 5467 & \text { PPARD } \\ \mathbf{3 5} & \mathbf{5 4 6 8} & \text { PPARG } \\ 36 & 6506 & \text { SLC1A2 } \\ \mathbf{3 7} & \mathbf{6 5 1 7} & \text { SLC2A4 } \\ 38 & 7019 & \text { TFAM }\end{array}$

Cell cycle/cell death/apoptosis/immune response

$\begin{array}{lll}\mathbf{3 9} & \mathbf{3 6 6} & \text { AQP9 } \\ 40 & 581 & B A X \\ 41 & 598 & B C L 2 L 1 \\ \mathbf{4 2} & \mathbf{6 2 7} & \boldsymbol{B D N F} \\ 43 & 834 & C A S P 1 \\ 44 & 836 & C A S P 3\end{array}$

Peroxisome proliferator-activated receptor gamma, coactivator 1 alpha

Peroxisome proliferator-activated receptor gamma, coactivator-related 1

Peroxisome proliferator-activated receptor alpha

Peroxisome proliferator-activated receptor delta

Peroxisome proliferator-activated receptor gamma

Solute carrier family 1 (glial high affinity glutamate transporter), member 2

Solute carrier family 2 (facilitated glucose transporter), member 4

Transcription factor A, mitochondrial

Aquaporin 9

BCL2-associated $\mathrm{X}$ protein

BCL2-like 1

Brain-derived neurotrophic factor

Caspase 1, apoptosis-related cysteine peptidase (interleukin 1, beta, convertase)

Caspase 3, apoptosis-related cysteine peptidase 
Table 2 (continued)

\begin{tabular}{|c|c|c|c|}
\hline & Gene ID & Gene Symbol & Function \\
\hline 45 & 839 & CASP6 & Caspase 6, apoptosis-related cysteine peptidase \\
\hline 46 & 841 & CASP8 & Caspase 8, apoptosis-related cysteine peptidase \\
\hline 47 & 1020 & $C D K 5$ & Cyclin-dependent kinase 5 \\
\hline 48 & 1211 & CLTA & Clathrin, light chain A \\
\hline 49 & 1213 & CLTC & Clathrin, heavy chain $(\mathrm{Hc})$ \\
\hline 50 & 3309 & HSPA5 & Heat shock 70 kDa protein 5 (glucose-regulated protein, 78 kDa) \\
\hline 51 & 3329 & HSPD1 & Heat shock 60 kDa protein 1 (chaperonin) \\
\hline 52 & 8870 & IER3 & Immediate early response 3 \\
\hline 53 & 9927 & MFN2 & Mitofusin 2 \\
\hline 54 & 27030 & MLH3 & MutL homolog 3 \\
\hline 55 & 2475 & MTOR & Mechanistic target of rapamycin (serine/threonine kinase) \\
\hline 56 & 4792 & NFKBIA & Nuclear factor of kappa light polypeptide gene enhancer in B-cells inhibitor, alpha \\
\hline 57 & 5921 & $R A S A 1$ & RAS p21 protein activator (GTPase activating protein) 1 \\
\hline 58 & 8767 & RIPK2 & Receptor-interacting serine-threonine kinase 2 \\
\hline 59 & 7124 & $T N F-a$ & Tumor necrosis factor (TNF superfamily, member 2) \\
\hline 60 & 608 & TNFRSF17 & Tumor necrosis factor receptor superfamily, member 17 \\
\hline 61 & 7157 & TP53 & Tumor protein $\mathrm{p} 53$ \\
\hline 62 & 7316 & $U B C$ & Ubiquitin $\mathrm{C}$ \\
\hline 63 & 3093 & $U B E 2 K$ & Ubiquitin-conjugating enzyme E2K (UBC1 homolog, yeast) \\
\hline 64 & 7345 & UCHL1 & Ubiquitin carboxyl-terminal esterase L1 (ubiquitin thiolesterase) \\
\hline \multicolumn{4}{|c|}{ Cell stress/Immune response } \\
\hline 65 & 875 & $C B S$ & Cystathionine-beta-synthase \\
\hline 66 & 948 & CD36 & CD36 molecule (thrombospondin receptor) \\
\hline 67 & 1639 & DCTN1 & Dynactin 1 (p150, glued homolog, Drosophila) \\
\hline 68 & 1843 & DUSP1 & Dual specificity phosphatase 1 \\
\hline 69 & 3117 & $H L A-D Q A 1$ & Major histocompatibility complex, class II, DQ alpha 1 \\
\hline 70 & 3576 & IL8 & Interleukin 8 \\
\hline 71 & 3553 & $I L 1 B$ & Interleukin 1 , beta \\
\hline 72 & 8564 & $K M O$ & KMO kynurenine 3-monooxygenase (kynurenine 3-hydroxylase) \\
\hline 73 & 6648 & SOD2 & Manganese superoxide dismutase \\
\hline \multicolumn{4}{|c|}{ Other transcription factors } \\
\hline 74 & 1386 & $A T F 2$ & Activating transcription factor 2 \\
\hline 75 & 1387 & CREBBP & CREB binding protein \\
\hline 76 & 1958 & EGR1 & Early growth response 1 \\
\hline 77 & 9611 & NCOR1 & Nuclear receptor co-repressor 1 \\
\hline 78 & 283131 & NEAT1 & Nuclear paraspeckle assembly transcript 1 (non-protein coding) \\
\hline 79 & 4899 & $N R F 1$ & Nuclear respiratory factor 1 \\
\hline 80 & 56731 & $S L C 2 A 4 R G$ & SLC2A4 regulator \\
\hline 81 & 6667 & $S P 1$ & Sp1 transcription factor \\
\hline 82 & 6874 & TAF4 & TAF4 RNA polymerase II, TATA box binding protein \\
\hline 83 & 6908 & $T B P$ & TATA box binding protein \\
\hline 84 & 7052 & $T G M 2$ & Transglutaminase 2 (C polypeptide, protein-glutamine-gamma-glutamyltransferase) \\
\hline 85 & 9322 & TRIP10 & Thyroid hormone receptor interactor 10 \\
\hline \multicolumn{4}{|c|}{ Calcium modulated proteins and HD related genes } \\
\hline 86 & 801 & CALM1 & Calmodulin 1 (phosphorylase kinase, delta) \\
\hline 87 & 805 & CALM2 & Calmodulin 2 (phosphorylase kinase, delta) \\
\hline 88 & 808 & CALM3 & Calmodulin 3 (phosphorylase kinase, delta) \\
\hline 89 & 51806 & CALML5 & Calmodulin-like 5 \\
\hline 90 & 9001 & $H A P 1$ & Huntingtin-associated protein 1 \\
\hline
\end{tabular}


Table 2 (continued)

\begin{tabular}{llll}
\hline & Gene ID & Gene Symbol & Function \\
\hline $\mathbf{9 1}$ & $\mathbf{3 0 9 2}$ & HIP1 & Huntingtin interacting protein 1 \\
92 & 3064 & HTT & Huntingtin \\
Preliminary normalizers and genomic DNA control & \\
93 & 3251 & HPRT1 & \\
94 & 60 & ACTB & \\
95 & 100008588 & 18S rRNA & \\
96 & & gDNA control & \\
\hline
\end{tabular}

${ }^{\text {a }}$ Statistically significant gene transcripts changes obtained in this study were distinguished by the bold type

Gene expression

In order to identify the transcription level changes between HDG and $\mathrm{CG}$ three types of comparisons were performed in this study, the first, between undifferentiated HDG and $\mathrm{CG}$, and the second, between HDG- and CG-cohorts, were both differentiated according to gender, age, BMI, calf circumference (CC) and Mini Nutritional Assessment (MNA). In addition, HDG-cohorts differentiated according to $\mathrm{CAG}$ number repeats, TFC, UHDRS and HD duration were compared with undifferentiated CG. The first transcript levels measurement in undifferentiated HDG $(n=29)$ and CG $(n=$ $28)$ revealed a statistically significant GPR FCs $(p<0.05)$ for MAOB (3.07), TGM2 (1.8), SLC2A4 (1.64) BCKDK (1.34), LDHA (-1.16) and BDNF (-2.11) (Fig. 1).

The HDG and CG were stratified according to the following characteristics: gender, age, BMI, CC, MNA and gene transcription rates were compared. Significant changes in transcription were detected between the various sub-cohorts (Table 1a). Comparison of gender revealed significant FCs for 3 transcripts (Table 3a): CD36, MAOB and SLC2A4. In the HDG the FCs for CD36 and MAOB were significantly higher in women than in men $(1.1$ vs. $-1.78 ; p<0.05)$ and $(2.34$ vs. $4.32 ; p<0.05)$ respectively. This was in contrast to SLC2A4, where the FC was higher in men $(1.89$ vs. $1.15 ; p<0.05)$. The age sub-cohort comparison (Table $3 \mathrm{~b}$ ) between the HDG and CG based on the GPR analysis showed significant FCs in 16 genes. The FCs range across age1- to age3-cohorts showed a decrease of CREBBP, SLC2A4RG and an increase of HSPA5, ATP2A2 in HDG. These changes were found in all three HDG age subcohorts; however the FC ranges across the age sub-cohorts were not statistically significant. The transcription changes of IL8, MTOR, BCKDHA, BCKDHB, MLH3 and UCP2 genes found across the age cohorts presented a FCs decrease together with age increase. The increased level of mRNA transcripts concerned RASA1, ACO2, UBE2K, HSPD1, PPARG and CASP6 was also found in the age2-and age3-cohorts regarding the reduced level only in the age1-cohort.

The BMI-cohort was differentiated into four sub-cohorts (BMI1-4) based on the BMI score ranges and reflected the general classification of obesity (Table 1a). Due to the relatively small number of HD and control subjects collected in the BMI1- and BMI4-cohorts, for the FCs calculation the BMI1- and BMI2-cohorts and also BMI3- and BMI4cohorts were pooled. Analysis of transcription level changes revealed 4 transcripts with statistically significant GPR FCs including PPRC1, LDHA, SLC2A and MAOB (Table 3c). The simultaneous increase of PPRC1 and decrease of MAOB transcripts across the BMI-cohorts were statistically significant according to the Kruskal Wallis test and appeared together with the increase of BMI index value.

The CC-cohort (Table 3d) was differentiated based on the size of calf circumference reflecting an increase of the approximate muscular mass amount into four $\mathrm{CC}$-cohorts (Table 1a.). A comparison of the qPCR results between the HDG and control yielded 5 transcripts FCs with $p<0.05$ based on GPR including an increase of IL8 transcript (from -2.48 for $\mathrm{CC} 1$ up to 1.41 for $\mathrm{CC} 3$ ), MAOB transcript (from 3.72 for $\mathrm{CC} 1$ up to 4.16 for $\mathrm{CC} 3$ ), and a decrease of $\mathrm{COX} 2$ transcript (from -1.05 for $\mathrm{CC} 1$ up to -1.27 for $\mathrm{CC} 3$ ), and $T G M$ transcript (from 2.62 for $\mathrm{CC} 1$ up to 1.36 for $\mathrm{CC} 4$ ), however $\mathrm{COX} 2 \mathrm{FC}$ ranges were not statistically significant.

The MNA-cohorts reflected a study group differentiation performed on the basis of the nutrition status assessment according to the MNA scale (Table 1a) (Rubenstein et al. 2001). Due to the relatively small number of healthy subjects found in the MNA1-cohort, control MNA1 and 2-cohorts (with MNA score 8-11) were pooled and used as a reference for the MNA1- and MNA2-cohort GPR FC calculations (Table 3e). The MNA-cohorts comparison between the HDG and control showed only two significant FCs, namely in $\mathrm{BDNF}$ and MAOB. The range of FC values for BDNF showed a transcript level decrease from -1.56 for the MNA1 to -2.55 for the MNA3-cohort and an increase of the MAOB level from 3.86 in the MNA1 up to 8.15 for MNA2, but a decrease of this gene transcript level in the MNA3 to 1.61.

The third type of the qPCR result analysis was performed for HDG sub-cohorts differentiated by the CAG number repeats, TFC, UHDRS motor scale assessment, the HD duration period and compared to no varied $\mathrm{CG}$ in order to 


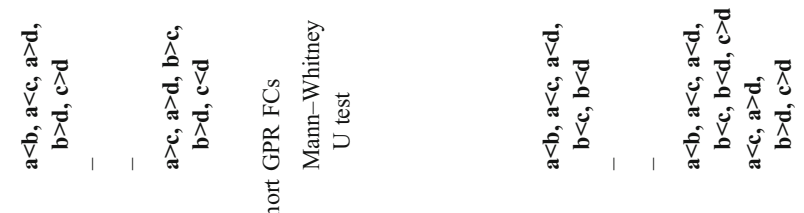

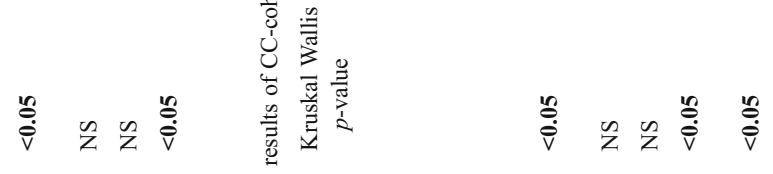

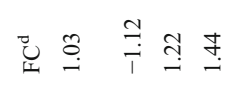

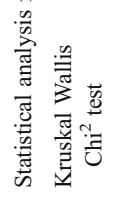

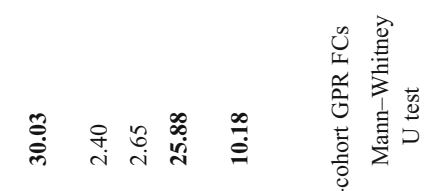

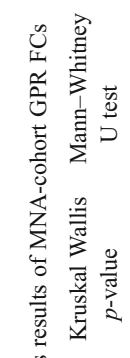

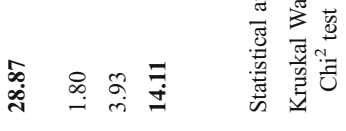

点

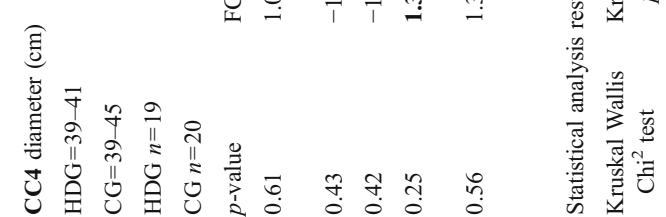

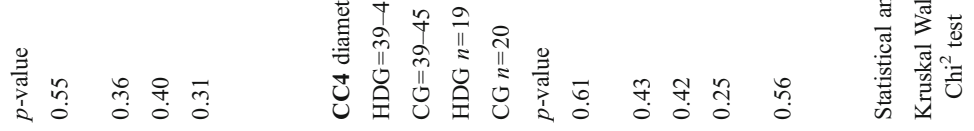

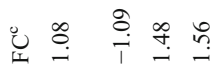

$$
\text { ¿ }
$$

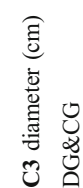

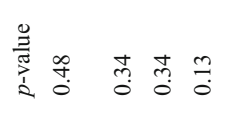

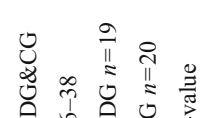

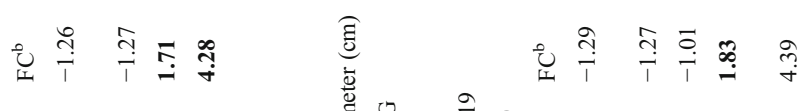

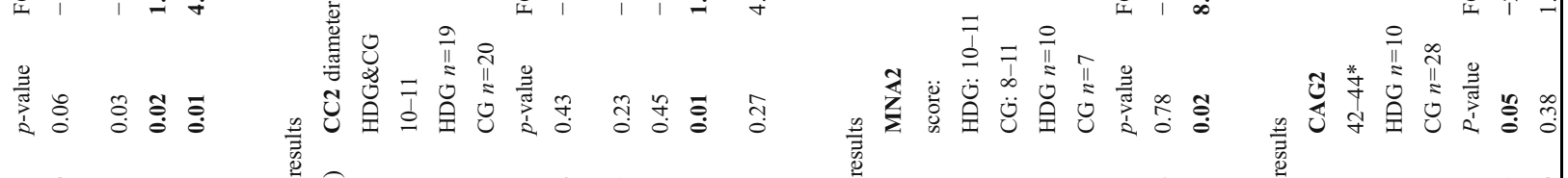

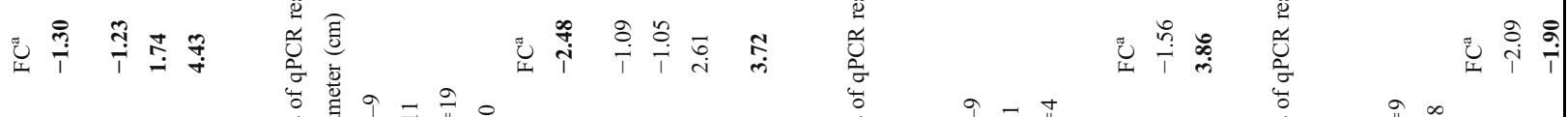

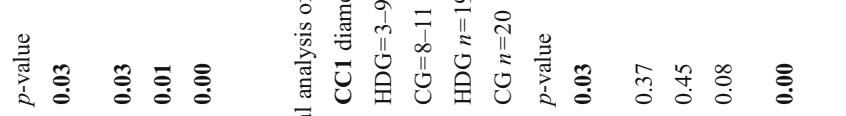

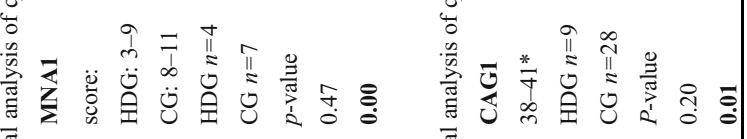

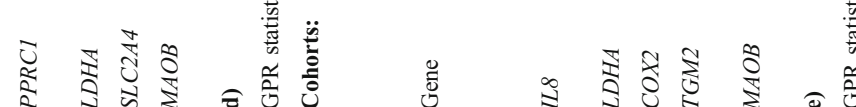
范

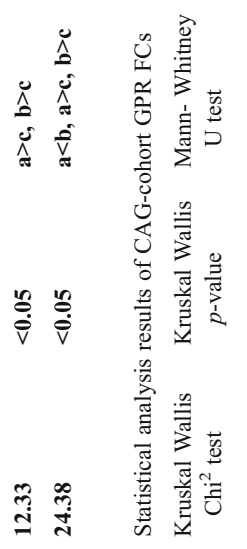

$\infty \stackrel{\circ}{\circ}$

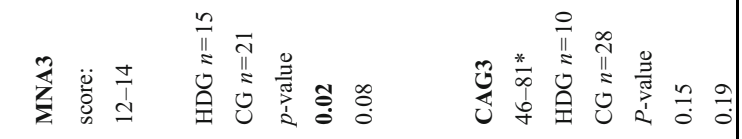
- $\quad$ 논 $\stackrel{\infty}{7} \frac{n}{\infty}$

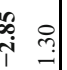




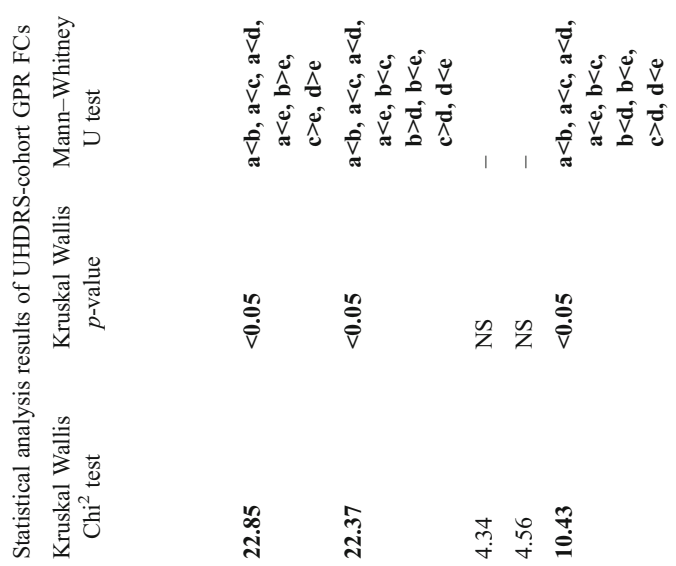

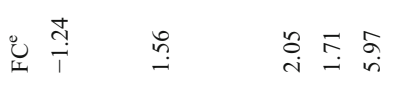

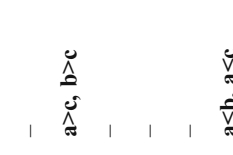

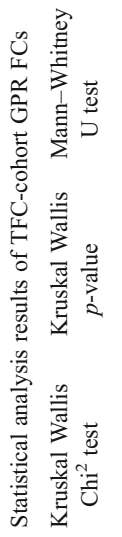

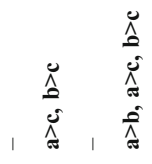

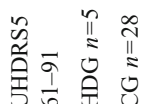

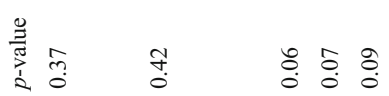

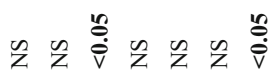

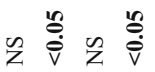

ت્己

$\exists \quad \stackrel{\infty}{T}$

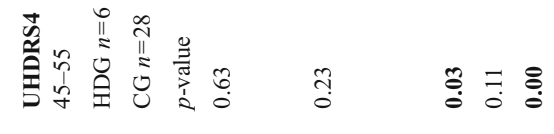

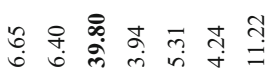

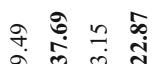

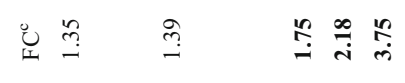

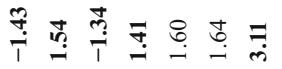

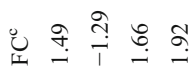

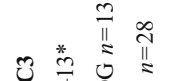

$\frac{\sqrt{2}}{3}$

$\frac{20}{2}$

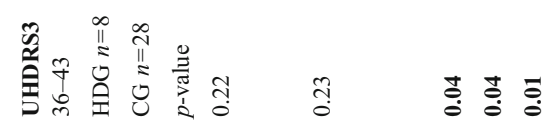

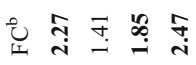

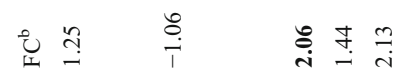

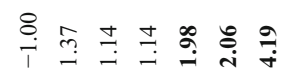

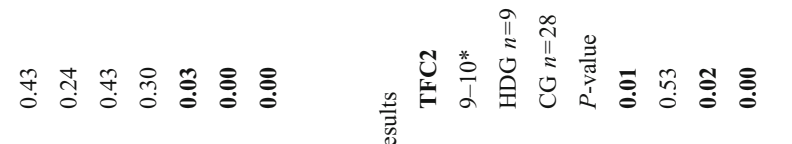

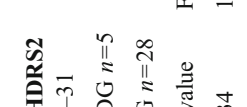

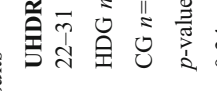

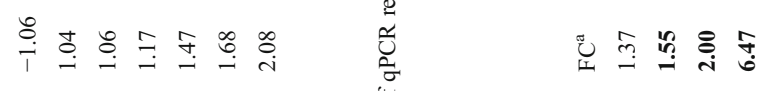

垔

$\stackrel{\infty}{i} \underset{T}{i}$

$\stackrel{\circ}{=} \stackrel{\infty}{-\infty}$

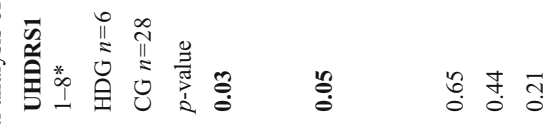

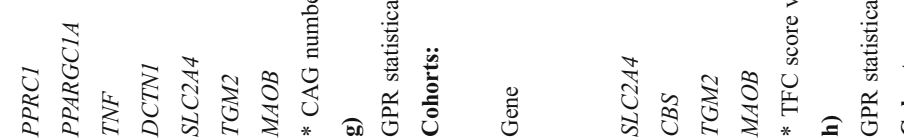
敦 


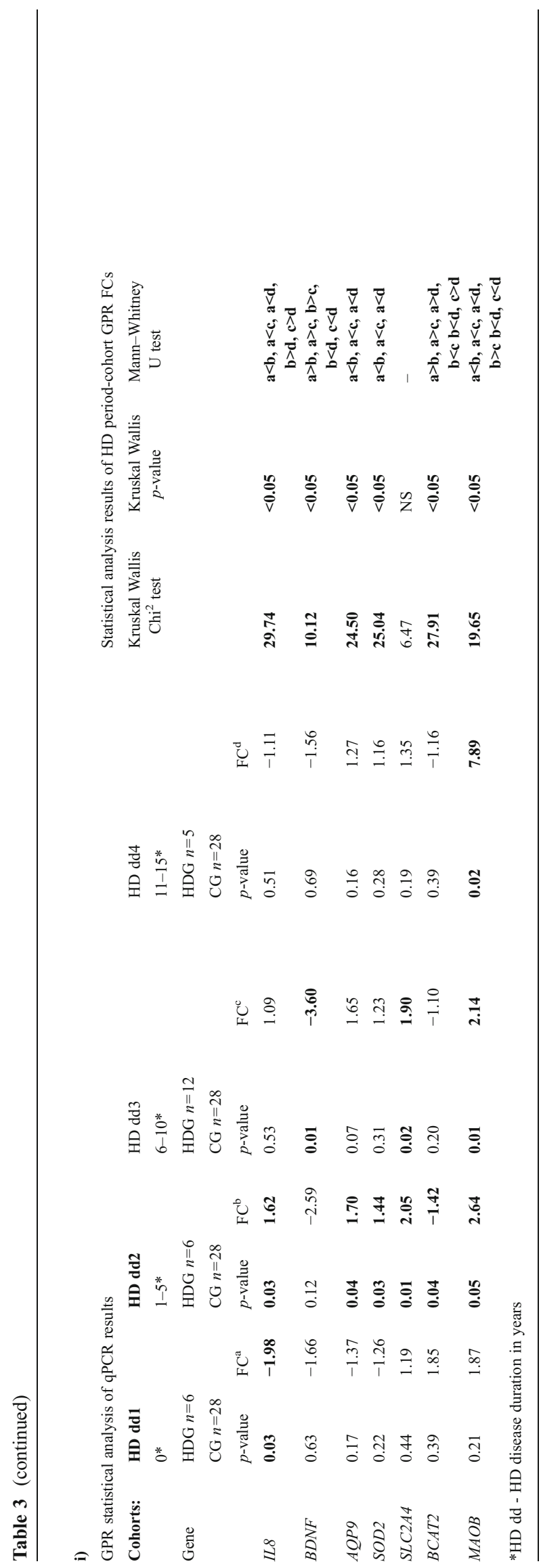

identify a panel of gene transcript changes related to the HD progress. Depending on the number of CAG repeats in larger allele of HTT gene, the HDG was divided into CAG1-3 cohorts (Table 1b). Significant FCs in 9 transcripts: BDNF, IL8, PPRC1, PPARGC1A, TNF- $\alpha$, DCTN1, SLC2A4, TGM2 and MAOB were observed (Table 3f). Across the CAG-cohorts, statistically significant FC range changes were found only for the IL8, TNF- $\alpha$ and MAOB transcripts, however, IL8 FC values simultaneously increased together with the CAG number repeats in CAG1and CAG2-cohorts. The TNF transcript levels presented almost the same level in the CAG1- and 2-cohorts (1.06 and 1.14 respectively), but significantly decreased up to -1.34 in the CAG3-cohort and the FC range was statistically significant. The increase of MAOB transcript level from 2.08 in the CAG1- into 4.19 in the CAG2- was simultaneously observed with the number of CAG repeats. The highest decrease of the BDNF FCs value was observed in the CAG2- $(-2.85)$ in contrast to the CAG1 cohort $(-2.09)$, however the BDNF FC range across the CAG1-3 cohorts was not statistically significant.

The TFC-cohorts reflected the stage of HD from functional ability perspective (Table 1b) and corresponded to HD progression. HD subjects with the most advanced functional decline were included in the TFC1-cohort (score: 1-8), the TFC2-cohort contains intermediate stage (score: 9-10) and the TFC3-cohort presymptomatic stage (score: 11-13). A comparative TFC-cohorts analysis (Table $3 \mathrm{~g}$ ) showed a statistically significant GPR FC concerning 4 transcripts (SLC2A4, CBS, TGM2 and MAOB). However Kruskal Wallis only confirmed significance for CBS and MAOB and showed the CBS FC decrease (from 1.55 in the TFC1- to -1.29 in the TFC3-cohort) and the MAOB FC decrease (from 6.47 in the TFC1- up to 1.92 in the TFC3-cohorts). The strongest increase of the CBS and MAOB FCs was found among HD subjects with the most advanced HD-stage (Table 3g).

Movement disorders may contribute to metabolic HD patients' status as well. Therefore UHDRS motor scale assessment was included in this analysis (Table 1b). The presymptomatic mutation carriers were located in the UHDRS1-cohort with the scores' range (1-8) what is an equivalent of mild motor symptoms. Subjects with most severe motor symptoms were included to the next UHDRS2-5-cohorts. Highest scored patients were included in the UHDRS5-cohort with the UHDRS motor scores from 61 to 91 . The FC results obtained for the UHDRS (1-5)-cohorts showed 5 transcripts with significant GPR FCs: IL8, HIP1, TGM2, SLC2A4 and MAOB. The Kruskal Wallis test confirmed significance in 3 FCs: IL8, HIP1 and MAOB (Table 3h).

The HD disease duration - cohort (dd) was differentiated into 5 sub-cohorts, the HD dd1-cohort consisting of presymptomatic carriers, the HD dd2-cohort (up to 5 years 
Table 4 Spearman's coefficient correlation in HDG and parameters related to Huntington disease

R- Spearman's coefficient correlation value; There are presented only statistically significant correlations

\begin{tabular}{|c|c|c|c|c|c|}
\hline \multirow[t]{2}{*}{ HD - sub-cohorts } & & \multicolumn{4}{|c|}{ HD - subjects } \\
\hline & & Age (years) & BMI- $\left(\mathrm{kg} / \mathrm{m}^{2}\right)$ & Calf circumference $(\mathrm{cm})$ & MNA -score \\
\hline \multirow[t]{2}{*}{ HD onset age -years } & $\mathrm{R}^{*}$ & 0.718 & 0.227 & -0.029 & 0.081 \\
\hline & $\mathrm{P}$ & $<0.01$ & 0.236 & 0.879 & 0.677 \\
\hline \multirow[t]{2}{*}{ CAG-repeats number } & $\mathrm{R}$ & -0.551 & -0.318 & -0.341 & -0.249 \\
\hline & $\mathrm{P}$ & 0.002 & 0.093 & 0.071 & 0.192 \\
\hline \multirow[t]{2}{*}{ TFC score } & $\mathrm{R}$ & -0.022 & 0.263 & 0.386 & 0.525 \\
\hline & $\mathrm{P}$ & 0.909 & 0.168 & 0.039 & 0.003 \\
\hline
\end{tabular}

from onset), HD dd3 (5-10 years from onset), HD dd4 (1015 years from onset), and HD dd5 (15-20 years from onset) respectively (Table 1b). The qPCR results comprised 7 transcripts with statistically significant FCs including IL8, BDNF, AQP9, SOD2, SLC2A4, BCAT2, and MAOB (Table 3i). The widest statistically significant FCs concerned BDNF (from -1.66 to -3.6 ) and BCAT2 (from 1.85 to -1.42 ) where a gradual decrease was observed and MAOB (from 1.87 to 7.89 ) where a gradual increase was observed following increase of HD duration.

To summarize, the first comparison results performed between undifferentiated HD- and Control Groups revealed 6 transcript genes, including the increase of MAOB, TGM2, SLC2A4, BCKDK and the decrease of LDHA and BDNF transcripts amount (Fig. 1) with statistically significant FC.
After sub-cohort comparisons 34 gene transcripts were distinguished (Table $3 \mathrm{a}-\mathrm{i}$ ), including genes involved in:

- energy metabolism: ACO2, ATP2A2, COX2, LDHA, MAOB, PPARG, PPARGC1A, PPRC1, UCP2;

- BCAA metabolism: BCAT2, BCKDHA, BCKDHB, BCKDK;

- other types of cellular processes: AQP9, CREBBP, BDNF, CASP6, CBS, CD36, DCTN1, HIP1, HSPA5, HSPD1, IL8, MLH3, MTOR, RASA1, SOD2, SLC2A4, SLC2A4RG, TGM2, TNF- $\alpha$ and UBE2K.

The widest FCs revealed in this study (Table $3 \mathrm{a}-\mathrm{i}$ ) concerned 7 transcripts including: MAOB (min. 1.44; max. 8.15), IL8 (min. -2.48; max. 1.41), HIP1 (min. -1.66; max. 1.56), BDNF (min. -3.6; max.-1.28), SLC2A4 (min. 1.19;

Table 5 Spearman's coefficient correlation between analytical tests results determined for HDG and CG

\begin{tabular}{|c|c|c|c|c|c|c|c|c|c|}
\hline \multirow[t]{2}{*}{ Analytical tests } & & \multicolumn{4}{|c|}{ HDG - subjects } & \multicolumn{4}{|c|}{ CG - healthy subjects } \\
\hline & & $\begin{array}{l}\text { Age } \\
\text { (years) }\end{array}$ & $\begin{array}{l}\text { BMI } \\
\left(\mathrm{kg} / \mathrm{m}^{2}\right)\end{array}$ & Calf circumference $(\mathrm{cm})$ & MNA -score & $\begin{array}{l}\text { Age } \\
\text { (years) }\end{array}$ & $\begin{array}{l}\text { BMI } \\
\left(\mathrm{kg} / \mathrm{m}^{2}\right)\end{array}$ & Calf circumference $(\mathrm{cm})$ & MNA- score \\
\hline \multirow[t]{2}{*}{ WBC } & $\mathrm{R}^{*}$ & -0.054 & 0.163 & 0.209 & 0.135 & -0.213 & 0.213 & 0.378 & 0.055 \\
\hline & $\mathrm{p}$ & 0.781 & 0.398 & 0.276 & 0.485 & 0.276 & 0.277 & 0.047 & 0.781 \\
\hline \multirow[t]{2}{*}{$\mathrm{RBC}$} & $\mathrm{R}$ & 0.329 & 0.436 & 0.227 & 0.111 & 0.009 & 0.040 & 0.101 & 0.139 \\
\hline & $\mathrm{p}$ & 0.081 & 0.018 & 0.235 & 0.567 & 0.964 & 0.839 & 0.608 & 0.482 \\
\hline \multirow[t]{2}{*}{ HGB } & $\mathrm{R}$ & 0.322 & 0.506 & 0.361 & 0.251 & 0.008 & 0.065 & 0.166 & 0.166 \\
\hline & $\mathrm{p}$ & 0.088 & 0.005 & 0.055 & 0.189 & 0.967 & 0.741 & 0.400 & 0.399 \\
\hline \multirow[t]{2}{*}{$\mathrm{HCT}$} & $\mathrm{R}$ & 0.334 & 0.439 & 0.305 & 0.087 & 0.004 & -0.016 & 0.149 & 0.006 \\
\hline & $\mathrm{p}$ & 0.077 & 0.017 & 0.108 & 0.654 & 0.985 & 0.937 & 0.459 & 0.977 \\
\hline \multirow[t]{2}{*}{$\mathrm{MCHC}$} & $\mathrm{R}$ & 0.043 & 0.353 & 0.274 & 0.494 & 0.086 & 0.189 & 0.315 & 0.356 \\
\hline & $\mathrm{p}$ & 0.825 & 0.060 & 0.151 & 0.006 & 0.665 & 0.336 & 0.103 & 0.063 \\
\hline \multirow[t]{2}{*}{ Lymphocytes } & $\mathrm{R}$ & 0.031 & 0.220 & 0.116 & 0.440 & -0.234 & 0.103 & 0.294 & -0.091 \\
\hline & $\mathrm{p}$ & 0.875 & 0.252 & 0.549 & 0.017 & 0.231 & 0.601 & 0.130 & 0.646 \\
\hline \multirow[t]{2}{*}{ ESR } & $\mathrm{R}$ & 0.504 & 0.422 & 0.207 & 0.286 & 0.190 & 0.175 & -0.048 & -0.213 \\
\hline & $\mathrm{p}$ & 0.005 & 0.023 & 0.282 & 0.133 & 0.332 & 0.373 & 0.808 & 0.277 \\
\hline \multirow[t]{2}{*}{ CRP } & $\mathrm{R}$ & 0.230 & 0.511 & 0.331 & 0.151 & 0.079 & 0.408 & 0.361 & -0.044 \\
\hline & $\mathrm{p}$ & 0.229 & 0.005 & 0.080 & 0.433 & 0.690 & 0.031 & 0.059 & 0.823 \\
\hline
\end{tabular}

R- Spearman's coefficient correlation value; $p$ - value. There are presented only statistically significant correlations 


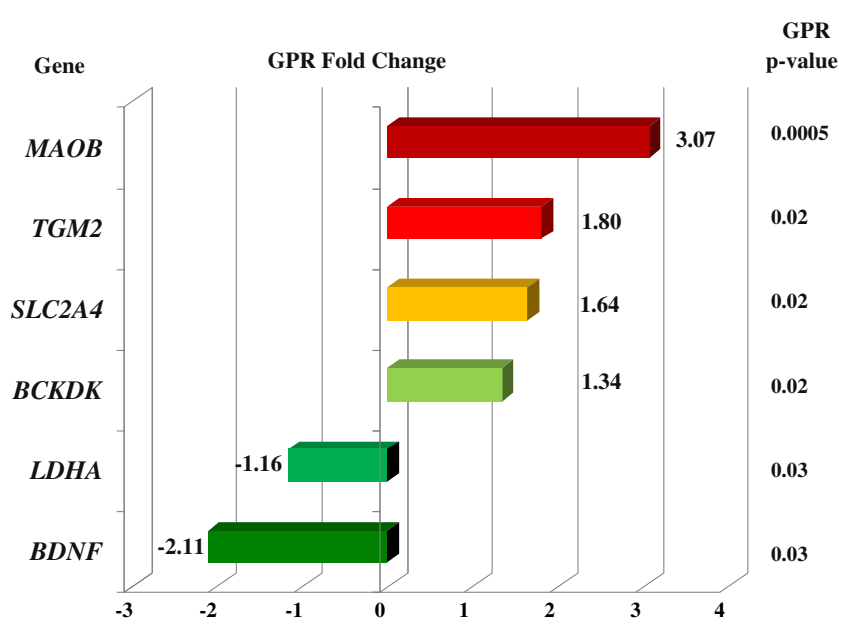

Fig. 1 A gene transcription level comparison between HDG $(n=29)$ and CG $(n=28)$ based on q-PCR results obtained by Global Pattern Recognition Software

max. 2.7), TGM2 (min. 1.36; max. 2.61), LDHA (min. -1.27 $\max .-1.09)$.

\section{Discussion}

The number of CAG repeats in the larger allele is responsible for up to $73 \%$ of the variability of HD onset age (Langbehn et al. 2004). It is therefore important to find other genetic and non-genetic factors such as gender, age, nutrition state, lifestyle, diet, activity or several etc. responsible for the remaining $27 \%$ of variability. This study was designed to identify possible metabolic biomarkers of $\mathrm{HD}$ at the gene transcription level. The relatively small study cohort limits the ability to achieve significant results; however, among the 95 genes tested, the transcription rate of 34 of them was significantly changed in the HDG. Statistically significant GPR FC values obtained after the comparison between undifferentiated HDG and CG revealed an increased level of four transcripts; MAOB, TGM2, SLC2A4 and BCKDK, and decreased levels of two transcripts; BDNF, LDHA, compared to healthy subjects.

The most significant transcription changes found in this study concerned the $M A O B$ gene encoding monoamine oxidase B. The MAOB protein is an outer mitochondrial membrane-bound enzyme that catalyzes the oxidative deamination of arylalkylamine neurotransmitters such as dopamine and serotonin and xenobiotic monoamines (Binda et al. 2003). In this study, the MAOB FC levels were the highest in the sub-cohorts of subjects with the most advanced HD level, such as the TFC1- (6.47), UHDRS motor5 (5.97) -cohorts, compared to the MAOB FC levels in the subcohorts of presymptomatic HD subjects such as TFC3(1.92) and UHDRS motor1 (1.87) -cohorts. .It also showed a gradual increase of the $M A O B$ gene transcript level in the course of HD duration from $\mathrm{FC}=1.87$ in the $\mathrm{HD}$ dd 1 up to $\mathrm{FC}=7.89$ in the $\mathrm{HD} \mathrm{dd} 4$. Interesting alterations were also found for the $M A O B$ gene transcript across the BMI-cohorts. The highest MAOB FC level was in the cohort of subject with the lowest $\mathrm{BMI}$ (BMI1-cohort, $\mathrm{FC}=4.43$ ) in contrast to the cohort with the highest BMI (BMI3-4 cohort, $\mathrm{FC}=1.44$ ). While this study is the first description of increased levels of the $M A O B$ gene transcript outside the CNS in HD-subjects, an abnormally high level of the MAOB activity has previously been demonstrated in the putamen, basal ganglia and other brain areas of HD subjects (Richards et al. 2011). Increased MAOB levels have also been described in the plasma of Alzheimer's disease patients, however $M A O B$ gene transcription was also increased in the brain. Increases of brain MAOB levels has also been shown in Parkinson's disease as well as in psychotics disorders, where it is accompanied by $M A O B$ polymorphism (Bergen et al. 2009; Emilsson et al. 2002; Naoi and Maruyama 2009). MAOB inhibitors are often clinically used in Parkinson's disease to improve motor function (Binda et al. 2003; Schapira 2010). In several neurodegenerative disorders, increased MAOB levels lead to neuronal damage caused by the neurotransmitters disturbances and an increase of the oxidative stress locally in specific brain regions (Bergen et al. 2009).

The MAOB protein location in the outer mitochondrial membrane can be related to a gradual increase of the $M A O B$ gene transcript level. Several mitochondrial abnormalities have been revealed in HD cells, including a decreased membrane resting potential, impaired calcium ion homeostasis, and marked morphological abnormalities with derangement of the mitochondrial matrix and cristae (Panov et al. 2002; Squitieri et al. 2006) and these mitochondrial abnormalities caused by mHTT can be recapitulated in normal lymphocytes treated with a fusion protein composed of a peptide containing a pathogenic polyglutamine tract (Panov et al. 2002). There is also evidence from HD mouse models (YAC72) and from cultured HD striatal neurons expressing endogenous mutant huntingtin (STHdhQ111) that the mHTT protein associates directly with the outer mitochondrial membrane (Choo et al. 2004). The direct relationship between the increase of the MAOB transcription levels in blood cells and its gradual increase concurrent with HD progression found in this study suggest a possible link with HD pathology.

The next significant changes found in this study concern the tissue transglutaminase 2 (TGM2, TG2). TGM2 is a calcium sensitive multifunctional enzyme with guanosine triphosphate signaling activity as well as transamidating activity (Munsie et al. 2011), and is present in the cytosol and nuclear fractions of the brain tissue (Cooper et al. 1999). It has previously been shown that the polyglutamine expansion in huntingtin results in cellular stresses including endoplasmatic reticulum disruption, increased calcium levels and activation of TGM2 resulting in aberrant cofilin-actin covalent cross- 
links (Munsie et al. 2011). In our study, an increase of the TGM2 gene transcription was found in a basic comparison and in some sub-cohorts analysis, including the CC-, CAG-, TFCand UHDRS-cohorts. An increased level of TGM2 was previously found in the HD brain and lymphocytes (Munsie et al. 2011), but in here that was confirmed only on the transcription level. The TGM2 FCs value alterations found in the subcohorts indicate a correlation between transcription changes and HD progress, indicated by an increase of TGM2 transcript level together with UHDRS-score and the number of CAG repeats, and with a decrease of the TFC-score. Additionally, the highest of TGM2 FCs values (2.61) was found in the CC1cohort with the smallest calf circumference diameter. Our data confirms that the quantifiable TGM2 gene transcription alterations in blood cells may be used as biomarkers for HD.

This study is the first to describe the increased level of the SLC2A4 transcription in HD subjects with reference to the control and also the transcript level increased across CAG1-2, TFC1-2, UHDRS1-3cohorts together with the HD progression (HD dd1-2). The SLC2A4 gene encodes an insulin-sensitive glucose transporter 4 (GLUT4) with a critical role in glucose homeostasis (Korgun et al. 2002). The clinical meaning of the SLC2A4 transcript alteration in the HD-progress is unknown so far and it needs further study, but it's potential as a biomarker in the monitoring of the HD progress outside the CNS could be useful.

The comparison between HDG and CG also showed the increase of the BCKDK (branched chain ketoacid dehydrogenase kinase) transcript level (1.34), while the sub-cohorts comparisons revealed the alteration of three other transcripts (BCKDHA, BCKDHB, and BCAT2) encoding enzymes involved in BCAA catabolism. BCKDK controls the catabolism of BCAA by branched-chain $\beta$-ketoacid dehydrogenase complex (BCKDC) regulation (Harris et al. 2004). An increase of the BCKDHB transcript level was found only in the age1-cohort (1.26), but across the age2-3-cohorts a decrease both of the BCKDHA and BCKDHB transcripts was found. The decrease of BCAA level and muscle mass, described previously in HD (Mochel et al. 2007), might be related also to the increased level of BCKDHA and BCKDHB ( $\alpha$-ketoacid dehydrogenase E1, alfa- and beta polypeptide). However the gene transcript changes involved in the BCAA metabolism haven't been found as statistically significant in the CC- and BMI-cohorts. The regulation of BCKDC is related to $\mathrm{BCKDK}$ and $\mathrm{BCKD}$ phosphatase (BCKDP) activity and linked to BCKDC level. Skeletal muscles are considered an initial site for BCAA catabolism because of a high activity of BCAA aminotransferase (BCAT2), a mitochondrial enzyme, responsible for the first step of BCAA catabolism (Brosnan and Brosnan 2006). In the HD dd1-cohort an increased level (1.85-fold) of the $B C A T 2$ gene transcript encoding aminotransferase was found, however in the HD dd2cohort this transcript level has been decreased to -1.42 -fold and has remained on the decreased level together with the disease duration. To clarify the BCAA metabolism disturbances in HD further studies are needed on the cellular level with cells derived from HD and healthy subjects.

The slight decrease of the LDHA gene transcript level found in this study $(-1.16)$ might reflect an impairment of glycolysis by inhibition of lactate acid conversion to pyruvate. The lactate level elevation due to the lactate dehydrogenase level decrease was previously described in the HD brain tissue and other tissues (Milakovic and Johnson 2005). A decrease of the LDHA gene transcript level was clearly expressed across the BMI- and CC- cohorts with the lowest LDHA transcript level (-1.23-fold) in the BMI1-cohort and the $\mathrm{CC} 2$-cohort. The decrease of these transcript levels seems to be deepening together with the lower BMI- and $\mathrm{CC}$-scores, reflected in lower body and muscle mass.

The strongest decrease found in this study concerned the $B D N F$ gene, encoding Brain-Derived Neurotrophic Factor protein and was obtained in a basic comparison between the $\mathrm{HD}$ and control as well as in the sub-cohorts. It was previously shown that the normal huntingtin protein positively influences the BDNF level, which protects and stimulates neuronal cells growth (Borrell-Pages et al. 2006). The strongest decrease in BDNF transcript levels was obtained in the CAG2-cohort (with 42-44 CAG repeats number) and in the dd-2-cohort. The reduction of the BDNF level had previously been described on a protein and transcript level in HD and also other neurodegenerative disorders, but a recent study confirms the phenomenon in HD only (Zuccato et al. 2011). Many contemporary studies indicate that mitochondrial dysfunction and oxidative stress play a crucial role in the majority of neurogenerative diseases. Mitochondria are the major source of intracellular reactive oxygen species and are particularly vulnerable to oxidative stress. In addition, the impairment of mitochondrial function disturbs the cellular energy homeostasis. PGC1 $\alpha$ stimulates the mitochondrial biogenesis and respiration, and this study has shown significant changes concerning two transcript levels, PPARGC1A and PPARC1, which are involved in energy metabolism. The FCs values showed a slight increase of PPARGC1A (1.54) and decrease of PPARC1 (-1.43) simultaneously with an increase of the number of CAG repeats. Additionally a decrease of the PPRC1 transcript level simultaneously occurred with the BMI decrease in the HDG when compared to the CG. These results are in line with the early energy deficits described in HD subjects, with the exception of PPARGC1A transcript levels which have been previously reported to decrease in HD (Strand et al. 2005; Weydt et al. 2006; Yoon et al. 2003). It is difficult to explain this phenomenon, but the HD-subjects who participated in this study had previously used a variety of diet supplements that might have influenced these gene transcripts that regulate energy metabolism on the transcription level. On the other 
hand, it has been proposed that aberrant transcriptional regulation of nuclear-encoded mitochondrial genes may be involved in HD pathogenesis.

The HD related metabolic changes suggested by this study need to be confirmed in a larger cohort before they can be considered HD specific biomarkers. The gene transcription level expressed by FCs and presented in this preliminary report indicates that it may be possible to select a HD biomarker from nuclear blood cells. Also, multiple analyses of gene transcript levels (sub-cohorts comparisons) showed more statistically significant FCs than the general comparison between the HDG and GC. This implies the multiple impact of particular genes on energy production and BCAA metabolism. The results of the study are promising both for the identification of biomarkers for HD and also for determining potential pharmacological targets for the improvement of these altered metabolic pathways.

Acknowledgments We are grateful to all the subjects who participated in the present study. This study was supported by European Huntington's Disease Network (Research project No, 0133).

Authors are also grateful to the Central Coordination EHDN for support in manuscript revision and thanks to Dr Anita Chadha-Patel for assistance with English.

Open Access This article is distributed under the terms of the Creative Commons Attribution License which permits any use, distribution, and reproduction in any medium, provided the original author(s) and the source are credited.

\section{References}

Akilesh S, Shaffer DJ, Roopenian D (2003) Genome Res 13:17191727. doi: $10.1101 /$ gr.533003

Atwal RS, Xia J, Pinchev D, Taylor J, Epand RM, Truant R (2007) Hum Mol Genet 16:2600-2615. doi:10.1093/hmg/ddm217

Beal MF, Brouillet E, Jenkins BG, Ferrante RJ, Kowall NW, Miller JM, Hyman BT (1993) J Neurosci 13:4181-4192

Bergen SE, Fanous AH, Walsh D, O’Neill FA, Kendler KS (2009) Schizophr Res 109:94-97

Binda C, Li M, Hubalek F, Restelli N, Edmondson DE, Mattevi A (2003) Proc Natl Acad Sci U S A 100:9750-9755. doi:10.1073/ pnas. 1633804100

Bjorkqvist M, Wild EJ, Thiele J, Silvestroni A, Andre R, Lahiri N, Tabrizi SJ (2008) J Exp Med 205:1869-1877

Borrell-Pages M, Canals JM, Cordelieres FP, Parker JA, Pineda JR, Grange G, Humbert S (2006) J Clin Invest 116:1410-1424. doi:10.1172/JCI27607

Brosnan JT, Brosnan ME (2006) J Nutr 136:207S-211S

Browne SE, Beal MF (2004) Neurochem Res 29:531-546

Chaturvedi RK, Calingasan NY, Yang L, Hennessey T, Johri A, Beal MF (2010) Hum Mol Genet 19:3190-3205

Choo YS, Johnson GV, MacDonald M, Detloff PJ, Lesort M (2004) Hum Mol Genet 13:1407-1420. doi:10.1093/hmg/ddh162
Cooper AJ, Sheu KF, Burke JR, Strittmatter WJ, Gentile V, Peluso G, Blass JP (1999) J Neurochem 72:889-899

Cui L, Jeong H, Borovecki F, Parkhurst CN, Tanese N, Krainc D (2006) Cell 127:59-69

Davies S, Ramsden DB (2001) Mol Pathol 54:409-413

Emilsson L, Saetre P, Balciuniene J, Castensson A, Cairns N, Jazin EE (2002) Neurosci Lett 326:56-60

Harris RA, Joshi M, Jeoung NH (2004) Biochem Biophys Res Commun 313:391-396

Korgun ET, Demir R, Sedlmayr P, Desoye G, Arikan GM, Puerstner P, Hahn T (2002) Blood Cells Mol Dis 28:152-159

Kosinski CM, Schlangen C, Gellerich FN, Gizatullina Z, Deschauer M, Schiefer J, Lindenberg KS (2007) Mov Disord 22:1637-1640. doi: $10.1002 / \mathrm{mds} .21550$

Kozlowski P, Sobczak K, Krzyzosiak WJ (2010) Genome Med 2:29

Langbehn DR, Brinkman RR, Falush D, Paulsen JS, Hayden MR (2004) Clin Genet 65:267-277. doi:10.1111/j.1399-0004.2004.00241.x

Liang H, Ward WF (2006) Adv Physiol Educ 30:45-151

Milakovic T, Johnson GV (2005) J Biol Chem 280:30773-30782

Mochel F, Charles P, Seguin F, Barritault J, Coussieu C, Perin L, Durr A (2007) PLoS One 2:e647. doi:10.1371/journal.pone. 0000647

Mochel F, Benaic S, Rabier D, Durr A (2011) Arch Neurol 68:265-267

Mochel F, Durant B, Meng X, O'Callaghan J, Yu H, Brouillet E, Durr A (2012) J Biol Chem 287:1361-1370

Munsie L, Caron N, Atwal RS, Marsden I, Wild EJ, Bamburg JR, Truant R (2011) Hum Mol Genet 20:937-1951

Naoi M, Maruyama W (2009) Expert Rev Neurother 9:1233-1250. doi:10.1586/ern.09.68

Panov AV, Gutekunst CA, Leavitt BR, Hayden MR, Burke JR, Strittmatter WJ, Greenamyre JT (2002) Nat Neurosci 5:731-736. doi: $10.1038 / \mathrm{nn} 884$

Perez-De La Cruz V, Santamaria A (2007) Physiol Res 56:513-526

Puigserver P, Rhee J, Donovan J, Walkey CJ, Yoon JC, Oriente F, Spiegelman BM (2003) Nature 423:550-555. doi:10.1038/ nature 01667

Richards G, Messe J, Waldvogel HJ, Gibbons HM, Dragunow M, Faull RL, Saura J (2011) Brain Res 1370:204-214

Ross CA, Tabrizi SJ (2011) Lancet Neurol 10:83-98

Rubenstein LZ, Harker JO, Salva A, Guigoz Y, Vellas B (2001) J Gerontol A Biol Sci Med Sci 56:M366-M372

Sathasivam K, Hobbs C, Turmaine M, Mangiarini L, Mahal A, Bertaux F, Bates GP (1999) Hum Mol Genet 8:813-822

Schapira AH (2010) Expert Opin Pharmacother 11:2261-2268. doi:10.1517/14656566.2010.511612

Squitieri F, Cannella M, Sgarbi G, Maglione V, Falleni A, Lenzi P, Fornai F (2006) Mech Ageing Dev 127:217-220

St-Pierre J, Drori S, Uldry M, Silvaggi JM, Rhee J, Jager S, Spiegelman BM (2006) Cell 127:397-408

Strand AD, Aragaki AK, Shaw D, Bird T, Holton J, Turner C, Olson JM (2005) Hum Mol Genet 14:1863-1876

The Huntington's Disease Collaborative Research Group (1993) Cell 72:971-983

Weydt P, Pineda VV, Torrence AE, Libby RT, Satterfield TF, Lazarowski ER, La Spada AR (2006) Cell Metab 4:349-362

Yoon JC, Xu G, Deeney JT, Yang SN, Rhee J, Puigserver P, Spiegelman BM (2003) Dev Cell 5:73-83

Zuccato C, Marullo M, Vitali B, Tarditi A, Mariotti C, Valenza M, Cattaneo E (2011) PLoS One. doi:10.1371/journal.pone.0022966 